\title{
The 4th IBIS/ISGRI survey and multi-wavelength follow-up program
}

\author{
Antony Bird*i \\ University of Southampton, Highfield, Southampton, SO17 1BJ, UK \\ E-mail: ajbirdesoton.ac.uk
}

\section{Nicola Masetti}

INAF - Istituto di Astrofisica Spaziale e Fisica Cosmica di Bologna, via Gobetti 101, 40129

Bologna, Italy

The INTEGRAL mission has produced a large number of detections of new sources during 7 years of operations. A considerable fraction of these sources are new detections at X-ray energies, and thus initially of unidentified nature. The sources that have been identified are described, and the efforts to identify and characterise the remaining sources are discussed. Possible strategies to maximise source discovery during future telescope operations are also discussed.

8th INTEGRAL Workshop "The Restless Gamma-ray Universe"

September 27-30 2010

Dublin Castle, Dublin, Ireland

\footnotetext{
* Speaker.

$\dagger$ Based on observations with INTEGRAL, an ESA project with instruments and science data centre funded by ESA member states (especially the PI countries: Denmark, France, Germany, Italy, Switzerland, and Spain), the Czech Republic and Poland, and with the participation of Russia and the US.
} 


\section{Introduction}

Since its launch in October 2002, the INTEGRAL mission has produced a large number of hard X-ray detections of new sources in 8 years of operations. A considerable fraction of these sources are new detections at X-ray energies, and thus initially of unidentified nature. The vast majority of new source detections have been made with the IBIS telescope which is capable of locating the source of emission with typically $4-5^{\prime}$ accuracy for a faint source. In some cases a clear $\mathrm{X}$-ray counterpart is already known, and if that source is located by Chandra, XMM or Swift/XRT, there is a good chance of immediately identifying one or more candidate optical/IR counterparts to the hard X-ray source. Very often this information, together with the X-ray characteristics of the source, leads to a rapid identification and characterisation of the class of emitter involved.

However, in a considerable fraction of cases, either there is no X-ray counterpart known, or for a variety of reasons the nature of the X-ray counterpart is still unknown. In this case, there is much work to do to identify the nature of the newly discovered source. The first stage of the process is to obtain a more accurate source location using an X-ray telescope. In the case of transient sources, this must be done at the earliest possible opportunity, and many observers now have X-ray TOO plans in place for just such a situation. After localisation of the source of the X-rays, a further stage of follow-up may be required in the form of optical/NIR spectroscopy.

This paper reviews the numbers of unidentified sources discovered so far, and the progress that has been made towards identifying their nature.

\section{The catalogs}

Since the purpose of this analysis is to understand how the number of unidentified sources have evolved during INTEGRAL's lifetime, it is most straightforward to use the four published 'IBIS/ISGRI survey catalogs' that have been compiled using, where possible, a consistent analysis method [1, 2, 3, 4]. Other catalogs have been produced, but with differences in software, techniques or aims. The four IBIS/ISGRI catalogs represent the most self-consistent set of catalogs that can be used to chart the progress of source discovery by INTEGRAL. The basic data used in the four catalogs are presented in Table 1.

\begin{tabular}{|c|c|c|c|}
\hline Catalogue & Exposure (Ms) & Dates & Sources \\
\hline 1 & 5 & Feb 2003 - Oct 2003 & 120 \\
2 & 10 & Feb 2003 - June 2004 & 209 \\
3 & 40 & Feb 2003 - Apr 2006 & 421 \\
4 & 70 & Feb 2003 - Apr 2008 & 723 \\
\hline
\end{tabular}

Table 1: The first four IBIS/ISGRI survey catalogs

The catalogs represent a growing sky coverage in both breadth and depth - while exposure continues to accumulate on the Galactic Center and Bulge regions, more uniform coverage of the extragalactic sky is slowly becoming available. 


\section{The IGR and unidentified sources}

From the first survey catalog, INTEGRAL has been discovering new sources. The first catalog, containing 123 sources, consisted of approx 60 sources that had been known from the early days of $\mathrm{X}$-ray astronomy, up to and including the Uhuru catalogs. The remaining previously known sources had been discovered by a variety of missions, but by far the next largest contingent, 27 or $~ 22 \%$ were new INTEGRAL discoveries. In some ways, this was a surprising result, but encouraged the idea that INTEGRAL might be observing in a regime that was previously quite poorly covered.

We can discuss both the 'IGR' and unidentified sources, and these terms are often interchanged. But in fact they represent two entirely different types of object. An 'IGR' source is named as such because it was not previously seen in high energy (X-ray and above) observations. Once a source has an IGR designation, it keeps it, and this forever labels it as an INTEGRAL discovery. Unidentified (or better, unclassified) sources are ones for which their nature is unknown. This represents a changing population, as new sources are discovered and are initially uncharacterised, but then eventually are studied in depth and are characterised as being of a particular class, at which point they are no longer unidentified sources. So we can learn quite different things by studying the evolution of IGR and unidentified source numbers.

Starting with the IGR sources, we would expect that as the sensitivity limits of the INTEGRAL surveys improve, more and more of the catalog sources will be INTEGRAL discoveries. The situation may well be complicated by the parallel operations of the Swift/BAT telescope which is also accumulating heavy exposure and achieving good sensitivity, especially in the extragalactic sky - but in fact we do indeed see a steady growth of the fraction of IGR sources in the IBIS surveys (Figure 1). While the first catalog contained 22\% IGRs, cat2 contained 58 'IGR' sources out of 209 (27\%), cat3 contained 168 'IGR' sources out of 421 (40\%) and now we can see that at least 52\% of the sources of the latest fourth catalog were discovered as X-ray emitters by INTEGRAL. In practice, the number is higher than this, because 'famous' sources retain their names in the catalog, even if first seen at X-ray energies by INTEGRAL.

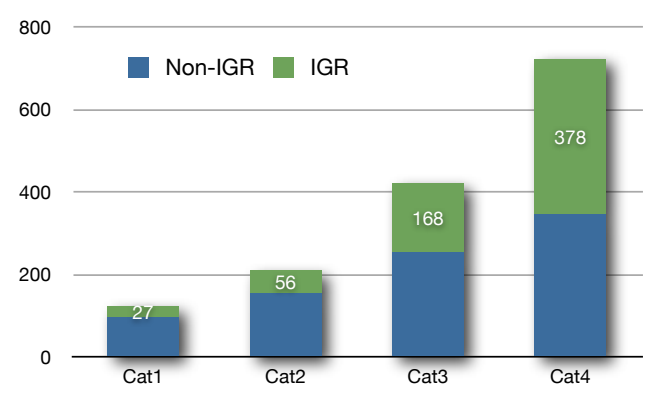

Figure 1: Evolution of 'IGR' numbers

As discussed previously, the situation for unidentified sources is less clear; as well as sources being identified, each catalog can also 'inherit' unidentified sources from a previous catalog. Four known sources seen in cat 1 had previously evaded attempts to determine their nature. That they 
are now all known is testament to the efforts made to identify the unknown sources, but also the additional diagnostic information provided by INTEGRAL that has enabled them to be understood.

By now, all but 2 of the unknown sources of cat 1 have been characterised. They were dominated by HMXB, LMXB, magnetic CVs and AGN, the large numbers of the former classes being due to the heavy exposure bias towards the galactic plane and bulge in early INTEGRAL operations. Prominent within these sources were the early discoveries of INTEGRAL - the obscured and highly transient supergiant systems not seen by previous missions. The situation was essentially repeated in cat2, where 32 of the 55 IGR sources were of unknown type at time of publication. Of these, 8 have now been identified as HMXB, 8 as AGN, 3 as CVs, 3 as LMXB, 2 as PWN/SNR and 7 are still unclassified. Considerably larger fractions of the unidentified sources in cat 3 and cat 4 are still being followed up (see next section).

Figure 2 shows how the source types has changed with each catalog.

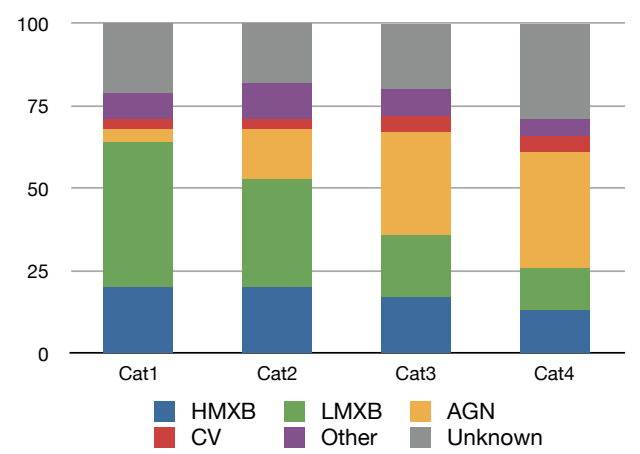

Figure 2: Evolution of source type percentages

At the time of publication, each catalog has contained 20-30\% of unidentified sources. Although the fraction may be increasing slightly, this does indicate that the programmes in place to identify sources are working hard to keep pace with the discovery rate, but in practice the number of unidentified sources is rising significantly. In order to understand why that is, let us now look at how these source identifications are being accomplished.

\section{The follow-up programme}

Follow-up of a new INTEGRAL source depends somewhat on how it was detected. For bright flaring sources detected in near real time during observations, rapid X-ray and multi-wavelength follow-ups are usually triggered via the Atel system, and the counterpart of the INTEGRAL source is usually quite obvious due to the flaring character of the source. However, many of the surveydiscovered sources may only be recognised after stacking of many Ms of data, and therefore they will often be faint persistent sources, unremarkable in other wavebands. Since the point source location accuracy (PSLA) for a faint INTEGRAL source may be as poor as $5^{\prime}$ error circle, this will lead to great source confusion in optical/IR sky images. Therefore the first stage of most source follow-ups is the identification of a soft X-ray counterpart with a better-defined position. At times, this can be achieved by correlation with other catalogs [5], or re-analysis of archival observations. 
On other occasions, dedicated X-ray observations can be performed to try to identify the X-ray counterparts of IBIS hard X-ray sources. Campaigns are underway by several teams using Chandra (see [6, 7] for examples), XMM [8], and Swift/XRT [9]. All these instruments have significantly better sensitivity than IBIS/ISGRI, and so there will often be multiple sources within the IBIS error circle. Nevertheless, if we also require that the X-ray source is visible at higher energies (typically above $3 \mathrm{keV}$ ) then the amount of ambiguity in source identification is much reduced. Other information, such as possible variability and source morphology can also be used to deduce the most likely X-ray counterpart.

Once an X-ray counterpart with a typical arc-second localisation has been identified, the next stage is to try to identify corresponding optical counterparts, and finally to obtain spectroscopic data on the most likely counterparts. Spectra have now been obtained on over 150 candidate sources $([10,11,12,13]$ for recent examples). Looking at the statistics of the sources identified so far, we can see that the follow-up programme has discovered a huge variety of sources types including: 11 LMXBs; 21 BeX binaries; 17 other HMXBs (often obscured); 96 nearby AGN (with a fairly even Sy1/Sy2 split); 4 XBONGs; 4 high-Z blazars; 3 BL Lacs; 19 CVs (mostly magnetic); 4 symbiotic stars and one active star.

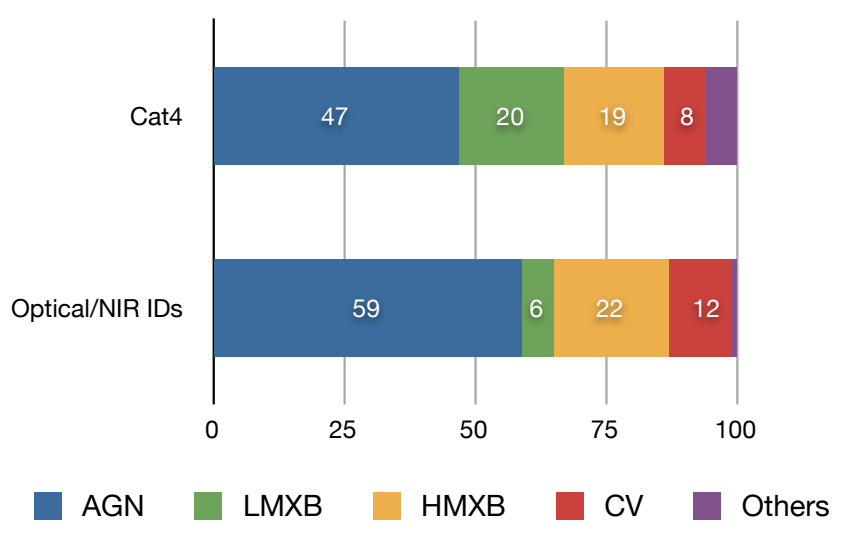

Figure 3: Percentages of source types in the fourth IBIS catalog and discovered in optical/NIR campaigns

Looking at the broader source categories, we can see that the populations being found in the optical/NIR searches are quite different to the overall catalog (Figure 3). This may be due to two factors. Firstly, there is undoubtedly an experimental bias in the selection of targets, concentrating on those away from the Galactic Plane, where the identification of counterparts is simpler - such an effect would increase the chances of finding the isotropic populations (AGN and CVs). Secondly, there is the fact that the sources being discovered in this way are by their nature previously unidentified - which implies that there is something intrinsically more difficult about their identification. In the case of the HMXBs being discovered, both intrinsic obscuration and high variability would have contributed to these sources being undetected until now.

\section{Discussion}

In conclusion, it is clear that the follow-up campaign to identify the previously unknown IN- 
TEGRAL sources has made outstanding progress. Nevertheless, the still increasing numbers of sources, and especially the new techniques discovering transient sources with unprecedented efficiency, mean that the work will continue for some time. The mostly persistent extragalactic sources are being identified with very high efficiency. Transient sources bring their own special problems, and prompt X-ray follow-up is essential to ensure unambiguous identification of counterparts in other wavebands in cases where the quiescent emission of the sources is low or even undetectable. Future observations with INTEGRAL in the Galactic Bulge and along the Galactic Plane will continue to uncover new transient sources, and the follow-up programmes will continue to be an essential part of the science exploitation of these new discoveries.

\section{References}

[1] A.J. Bird, et al., The First IBIS/ISGRI Soft Gamma-Ray Galactic Plane Survey Catalog, ApJ, 607, (2004), L33.

[2] A.J. Bird, et al., The Second IBIS/ISGRI Soft Gamma-ray Survey Catalog, ApJ, 636, (2006), 175.

[3] A.J. Bird, et al., The Third IBIS/ISGRI Soft Gamma-ray Survey Catalog, ApJ Suppl., 170, (2007), 175.

[4] A.J. Bird, et al., The Fourth IBIS/ISGRI Soft Gamma-ray Survey Catalog, ApJ Suppl., 186, (2010), 1.

[5] J.B. Stephen, Using the ROSAT Bright Source Catalogue to find counterparts for IBIS/ISGRI survey sources, A\&A, 432, (2005), L49

[6] J.A. Tomsick, et al., Chandra Localizations and Spectra of Integral Sources in the Galactic Plane: The Cycle 9 Sample, ApJ,701, (2009), 811

[7] M. Fiocchi et al., Five New INTEGRAL Unidentified Hard X-ray Sources Uncovered by Chandra, ApJ, 720, (2010), 987.

[8] Malizia, A., et al., XMM-Newton observations of unidentified INTEGRAL/IBIS sources, MNRAS, 408, 975.

[9] R. Landi, et al., Swift/XRT observations of unidentified INTEGRAL/IBIS sources, MNRAS, 403, (2010), 945

[10] I. Negueruela, et al., Optical and Infrared characterisation of High Mass X-ray Binaries discovered by INTEGRAL, in proceedings of 7th INTEGRAL Workshop, PoS (Integral 08 ) 072

[11] S. Chaty, et al., Multi-wavelength observations of Galactic hard X-ray sources discovered by INTEGRAL. I. The nature of the companion star, A\&A, 484, (2008), 783.

[12] N. Masetti, et al., Unveiling the nature of INTEGRAL objects through optical spectroscopy. VII. Identification of 20 Galactic and extragalactic hard X-ray sources, A\&A, 495, (2009), 121.

[13] N. Masetti et al., Unveiling the nature of INTEGRAL objects through optical spectroscopy. VIII. Identification of 44 newly detected hard $X$-ray sources, A\&A, 519, (2010), 96 